\title{
INTERPRETAÇÃO DE ÍNDICES DRIS PARA A CULTURA DO CUPUAÇU ${ }^{(1)}$
}

\author{
Paulo Guilherme Salvador Wadt( ${ }^{(2)}$, Jairo Rafael Machado Dias ${ }^{(3)}$, \\ Daniel Vidal Perez ${ }^{(4)}$ \& Cleigiane de Oliveira Lemos ${ }^{(2)}$
}

\begin{abstract}
RESUMO
A produtividade dos pomares de cupuaçu na Amazônia tem experimentado lento declínio, provavelmente devido à diminuição da fertilidade do solo, requerendo adubações. Na ausência de recomendações técnicas para quantificar a demanda por nutrientes por essa cultura, o uso do Sistema Integrado de Diagnose e Recomendação (DRIS) pode representar uma alternativa para suprir essa necessidade. $O$ objetivo deste trabalho foi avaliar os diagnósticos produzidos por diferentes fórmulas e critérios de interpretação dos índices DRIS em pomares de cupuaçu. Amostras foliares de 153 pomares foram analisadas para os nutrientes N, P, K, Ca, Mg, Zn, Mn, Cu e Fe e diagnosticadas por três fórmulas DRIS: Beaufils, Jones e Wadt e colaboradores. Na interpretação dos índices DRIS, utilizaram-se: o método DRIS Matéria Seca (M-DRIS), que separa os nutrientes em limitantes ou não limitantes, e o método do Potencial de Resposta à Adubação, o qual separa os nutrientes no estado nutricional de insuficientes, equilibrados ou em excesso. Foram também adotados níveis críticos para os teores dos nutrientes foliares em cupuaçueiros, oriundos da literatura. Os diagnósticos produzidos pelos diferentes critérios de interpretação dos índices DRIS não foram consistentes entre si ou em comparação com o método do nível crítico. A exceção foi a fórmula de Wadt e colaboradores que mostrou maior consistência entre os diagnósticos, independentemente do critério utilizado para sua interpretação. Os valores sugeridos para avaliação do estado nutricional pelo método do nível crítico não foram satisfatórios.
\end{abstract}

Termos para indexação: estado nutricional, diagnose foliar, Amazônia, fruteiras, Theobroma grandiflorum.

(1) Recebido para publicação em 20 de maio de 2010 e aprovado em 19 de outubro de 2011.

(2) Pesquisador, Analista, Embrapa Acre. Caixa Postal 321, CEP 69908-970 Rio Branco (AC). E-mails: paulo@cpafac.embrapa.br; cleigiane@cpafac.embrapa.br

(3) Professor, Universidade Federal de Rondônia. Avenida Norte Sul 7300, CEP 76940-000 Rolim de Moura (RO). E-mail: jairorafaelmdias@hotmail.com

(4) Pesquisador, Embrapa Solos. Rua Jardim Botânico 1.024. CEP 22460-000 Rio de Janeiro (RJ). E-mail: daniel@cnps.embrapa.br 


\title{
SUMMARY: DRIS INTERPRETATION CRITERIA FOR CUPUASSU OR- CHARDS
}

\begin{abstract}
The productivity of cupuassu orchards in the Amazon region has been gradually declining, probably due to the reduction in soil fertility, calling for fertilization. In the absence of technical recommendations to calculate the nutrient demand of an orchard, the use of the Integrated Diagnosis and Recommendation (DRIS) may represent an alternative to fill this gap. The purpose of this study was to evaluate the diagnoses based on different formulas and criteria of interpretation of DRIS indices of cupuassu orchards. The contents of $N, P, K, C a$, $\mathrm{Mg}, \mathrm{Zn}, \mathrm{Mn}, \mathrm{Cu}$, and $\mathrm{Fe}$ were determined in leaf samples from 153 orchards and diagnosed by three DRIS formulas: Beaufils, Jones and Wadt and coleagues. The DRIS indices were interpreted by: DRIS including dry matter (M-DRIS), which separates the nutrients in limiting or non-limiting, and the method of the response potential to fertilization, which classifies the nutritional status of elements in insufficient, balanced or excess. Critical levels of foliar nutrients were also adopted for cupuassu trees from the literature. The diagnoses based on the different interpretation criteria of the DRIS indices were not consistent with each other or with the threshold method, except for the formula Wadt and coleagues. The diagnoses by the Wadt and coleagues formula were more consistent, regardless of the criteria used for interpretation. The suggested values to assess the nutritional status by the threshold method were not satisfactory.
\end{abstract}

Index terms: nutritional status, leaf analysis, Amazon region, fruit trees, Theobroma grandiflorum.

\section{INTRODUÇÃO}

O cultivo de cupuaçueiros na Amazônia encontrase em fase de domesticação, com destacado potencial econômico e social. Os pomares de cupuaçueiros apresentam produtividade extremamente variável, sofrendo declínio na produtividade nos últimos 10 anos em algumas áreas da Amazônia (Alfaia \& Ayres, 2004). No projeto de Reflorestamento Econômico Consorciado Adensado (RECA), localizado no distrito de Vila Califórnia, em Rondônia, os principais fatores responsáveis pelo declínio na produtividade foram a broca dos frutos e a vassoura de bruxa (Lopes \& Silva, 1998) e a baixa fertilidade natural dos solos associada às altas taxas de exportação de nutrientes pela cultura (Alfaia et al., 2004).

Nesse sentido, a partir de uma produtividade média de $7.900 \mathrm{~kg} \mathrm{ha}^{-1}$ de frutos, as exportações podem atingir até $54 \mathrm{~kg} \mathrm{ha}^{-1}$ de $\mathrm{K}, 48 \mathrm{~kg} \mathrm{ha}^{-1}$ de $\mathrm{N}, 8 \mathrm{~kg} \mathrm{ha}{ }^{-1}$ de Ca, $8 \mathrm{~kg} \mathrm{ha}^{-1} \mathrm{de} \mathrm{Mg}$ e $6 \mathrm{~kg} \mathrm{ha}^{-1}$ de $\mathrm{P}$, valores estimados a partir de sua exportação pelos frutos de cupuaçu, cultivados em Gleissolos e Latossolos da Amazônia (Costa, 2006). Embora a resposta a $\mathrm{K}$ tenha sido linear a doses de 0,80 e $160 \mathrm{~kg} \mathrm{ha}^{-1}$ de $\mathrm{K}_{2} \mathrm{O}$, em um ensaio conduzido em um Argissolo Vermelho-Amarelo, e ausência de resposta a $\mathrm{N}$ e $\mathrm{P}$, a maior produtividade foi obtida na aplicação de 60-100-80 $\mathrm{kg} \mathrm{ha}^{-1}$, respectivamente de $\mathrm{N}, \mathrm{P}_{2} \mathrm{O}_{5}$ e $\mathrm{K}_{2} \mathrm{O}$, sugerindo o efeito benéfico da aplicação de $\mathrm{N}$ e $\mathrm{P}$ na dose intermediária de $\mathrm{K}$ (Alfaia \& Ayres, 2004).

Uma alternativa para aumentar a produtividade de plantas cultivadas está na melhoria do seu estado nutricional, aumentando-se a disponibilidade dos nutrientes com maior grau de deficiência. A interpretação do estado nutricional da planta, com base na análise química dos tecidos foliares, pode ser feita tanto pelo nível crítico (NC) como pela Faixa de Suficiência, que são os métodos tradicionais, ou ainda pelo sistema DRIS (Diagnosis and Recommendation Integrated System - Beaufils, 1973); este último tem a vantagem considerar o equilíbrio nutricional (Mourão Filho, 2002).

Embora o método DRIS tenha se mostrado promissor para muitas culturas, ainda persistem controvérsias a respeito dos procedimentos indicados no cálculo das normas e dos índices DRIS (Mourão Filho, 2002), como, por exemplo, o uso das fórmulas mais simples (Jones, 1981) ou de soluções mais complexas, como a fórmula de Wadt et al. (2007). Acrescenta-se ainda que os critérios para interpretação dos índices DRIS também não estão claros. Há proposições unicamente em função de o índice ser negativo ou positivo, indicando insuficiência e excesso, respectivamente (Mourão Filho, 2002), ou a utilização do DRIS de Matéria Seca (M-DRIS), o qual separa os nutrientes entre limitantes e não limitantes, comparando-se o valor do índice DRIS de cada nutriente com o índice DRIS de matéria seca (Hallmark et al., 1987) e, também, com o critério do Potencial de Resposta à Adubação - PRA (Wadt, 2005).

O objetivo deste trabalho foi avaliar os diagnósticos produzidos por diferentes fórmulas e processos de interpretação dos índices DRIS, comparando-os entre si e com o critério do nível crítico. 


\section{MATERIAL E MÉTODOS}

Dada a inexistência de informações na literatura que permitissem a obtenção dos níveis críticos para os teores foliares dos nutrientes em pomares de cupuaçu, optou-se pela proposição de um critério baseado na extrapolação de dados experimentais, que foi denominado de nível crítico preliminar (NC).

O nível crítico preliminar (NC) foi definido pela expressão: $\mathrm{NC}=\mathrm{Vo}-[0,25(\mathrm{Vo}-\mathrm{Vd})]$, em que $\mathrm{Vd}$ corresponde ao nível de dano fisiológico para um dado nutriente e Vo ao valor ótimo para o mesmo nutriente, ambos em $\mathrm{g} \mathrm{kg}^{-1}$ de matéria seca. Os valores de Vo para N, P e K corresponderam aos teores médios do ensaio de adubação de maior produtividade, em árvores de cupuaçu de frutos com e sem sementes, conduzido por Alfaia \& Ayres (2004). Para Ca, Mg, Zn, Fe e Mn, os teores ótimos foram obtidos de Costa (2006), de pomares cultivados em Latossolos e Gleissolos. Para $\mathrm{Cu}$, foi adotado o valor ótimo determinado por Salvador et al. (1994), em ensaio de casa de vegetação, para folhas maduras (em mudas com 10 meses de idade). Quanto ao nível de dano visual (Vd), para todos os nutrientes, foram adotados os teores foliares correspondentes ao início do processo de dano visual em folhas maduras de mudas cultivadas em vasos (Salvador et al., 1994).

Neste estudo, as normas DRIS foram obtidas de monitoramento nutricional realizado entre julho e setembro de 2008, em 153 pomares comerciais de cupuaçueiro. O monitoramento consistiu na obtenção, em cada pomar, de 30 amostras da terceira folha de lançamento recém-amadurecido, a partir do ápice do ramo de altura média, de ramos localizados nas faces norte e sul das árvores amostradas (Costa, 2006).

Os pomares monitorados, com idade variando de 5 a 18 anos, foram cultivados em sistema de monocultivo (42 pomares) ou em sistemas agroflorestais (111 pomares) e localizam-se no distrito de Vila Califórnia, em um quadrante definido pelas coordenadas $9^{\circ} 32$ ' 39 a $58^{\prime \prime}$ sul e $66^{\circ} 16^{\prime} 32$ a $95^{\prime \prime}$ oeste e $9^{\circ} 53^{\prime} 41$ a $12^{\prime \prime}$ sul e $66^{\circ} 35^{\prime} 34$ a $60^{\prime \prime}$ oeste, no extremo oeste do município de Porto Velho-RO.

Em cada amostra foliar composta, foram determinados os teores dos nutrientes, por digestão nítrico-perclórica, para determinação dos teores de $\mathrm{Ca}, \mathrm{Mg}, \mathrm{Mn}, \mathrm{Fe}$, $\mathrm{Zn}$ e $\mathrm{Cu}$ por espectrometria de plasma (ICP-OES); de K, por fotometria de chama; de $\mathrm{P}$, por espectrofotometria molecular; e de $\mathrm{N}$ total, por digestão sulfúrica a destilação pelo método Kjehdahl, conforme protocolo descrito por Carmo et al. (2000).

Para as normas DRIS, foram obtidas as médias e o desvio-padrão amostral dos teores dos nutrientes e as relações entre os teores de dois nutrientes, após a transformação logarítmica dos dados (Beverly, 1987; Dias et al., 2009).

O procedimento utilizado na obtenção das normas DRIS a partir de toda a população foi realizado conforme proposto por Beverly (1987). Segundo esse autor, com o aumento do tamanho da amostra, todas as médias das relações nutricionais nas subpopulações tendem a um mesmo valor; portanto, aumentando-se o tamanho da amostra, melhor será a estimativa da média verdadeira. Esse procedimento foi adotado porque as informações para a classificação dos pomares em relação à produtividade não foram consideradas seguras.

Os índices DRIS foram calculados pelas fórmulas de Beaufils (1973), Jones (1981) e Wadt et al. (2007) - esta última, com modificações. Todas as relações utilizadas nessas fórmulas foram log-transformadas (Beverly et al., 1993) e expressas conforme sugerido por Maia (1999):

Quadro 1. Nível crítico preliminar ${ }^{(1)}$ de teores foliares de nutrientes para o cupuaçueiro, obtido a partir do valor ótimo e do valor de dano visual

\begin{tabular}{llrrr} 
Nutriente & Unidade & Valor ótimo (Vo) & Valor de dano visual (Vd) & Nível crítico preliminar (NC) \\
\hline $\mathrm{N}$ & $\mathrm{g} \mathrm{kg}^{-1}$ & 16,50 & 10,50 & 15,00 \\
$\mathrm{P}$ & $\mathrm{g} \mathrm{kg}^{-1}$ & 1,08 & 0,90 & 1,04 \\
$\mathrm{~K}$ & $\mathrm{~g} \mathrm{~kg}^{-1}$ & 6,42 & 2,20 & 5,37 \\
$\mathrm{Ca}$ & $\mathrm{g} \mathrm{kg}^{-1}$ & 7,22 & 1,20 & 5,72 \\
$\mathrm{Mg}$ & $\mathrm{g} \mathrm{kg}^{-1}$ & 2,33 & 0,60 & 1,90 \\
$\mathrm{Zn}$ & $\mathrm{mg} \mathrm{kg}-1$ & 19,00 & 10,00 & 16,75 \\
$\mathrm{Fe}$ & $\mathrm{mg} \mathrm{kg}-1$ & 86,00 & 25,00 & 70,75 \\
$\mathrm{Mn}$ & $\mathrm{mg} \mathrm{kg}-1$ & 184,00 & 6,00 & 139,50 \\
$\mathrm{Cu}$ & $\mathrm{mg} \mathrm{kg}-1$ & 3,00 & 2,00 & 2,75
\end{tabular}

(1) NC: Vo - [0,25 (Vo - Vd)]; adaptado de Ayres \& Alfaia (2004), Costa (2006) e Salvador et al. (1994). 
i) Fórmula de Beaufils (1973):

Quando $\log (\mathrm{A} / \mathrm{B})<\log (\mathrm{a} / \mathrm{b})$

$$
\begin{gathered}
\mathrm{f}(\mathrm{A} / \mathrm{B})=\left\{[\log (\mathrm{A} / \mathrm{B})-\log (\mathrm{a} / \mathrm{b})] / \sigma_{(\log (\mathrm{a} / \mathrm{b}))\}} \mathrm{x}\right. \\
{[\log (\mathrm{a} / \mathrm{b}) / \log (\mathrm{A} / \mathrm{B})]}
\end{gathered}
$$

Senão

$$
\mathrm{f}(\mathrm{A} / \mathrm{B})=\left\{[\log (\mathrm{A} / \mathrm{B})-\log (\mathrm{a} / \mathrm{b})] / \sigma_{(\log (\mathrm{a} / \mathrm{b}))}\right\}
$$

ii) Fórmula de Jones (1981):

$$
\mathrm{f}(\mathrm{A} / \mathrm{B})=\left\{[\log (\mathrm{A} / \mathrm{B})-\log (\mathrm{a} / \mathrm{b})] / \sigma_{(\log (\mathrm{a} / \mathrm{b}))}\right\}
$$

iii) Fórmula de Wadt et al. (2007):

Quando o elemento "A" forem os nutrientes N, P ou K:

$$
\begin{gathered}
\mathrm{f}(\mathrm{A} / \mathrm{B})=\mathrm{fk} \times\left\{[\log (\mathrm{A} / \mathrm{B})-\log (\mathrm{a} / \mathrm{b})] / \sigma_{(\log (\mathrm{a} / \mathrm{b}))\}}\right\} \mathrm{x} \\
{[\log (\mathrm{a} / \mathrm{b}) / \log (\mathrm{A} / \mathrm{B})]}
\end{gathered}
$$

Quando o elemento "A" forem os nutrientes Ca ou Mg:

$$
\begin{gathered}
\text { se } \log (\mathrm{A} / \mathrm{B})<\log (\mathrm{a} / \mathrm{b}) \text { e } \log (\mathrm{A} / \mathrm{B})>\log (\mathrm{a} / \mathrm{b}) / 2 \\
\mathrm{f}(\mathrm{A} / \mathrm{B})=\mathrm{fk} \times\left\{[\log (\mathrm{A} / \mathrm{B})-\log (\mathrm{a} / \mathrm{b})] / \sigma_{(\log (\mathrm{a} / \mathrm{b}))}\right\} \text { x } \\
{[\log (\mathrm{A} / \mathrm{B}) / \log (\mathrm{a} / \mathrm{b})]} \\
\text { se } \log (\mathrm{A} / \mathrm{B})<\log (\mathrm{a} / \mathrm{b}) \text { e } \log (\mathrm{A} / \mathrm{B})<=\log (\mathrm{a} / \mathrm{b}) / 2 \\
\mathrm{f}(\mathrm{A} / \mathrm{B})=\mathrm{fk} \times\left\{[\log (\mathrm{A} / \mathrm{B})-\log (\mathrm{a} / \mathrm{b})] / \sigma_{(\log (\mathrm{a} / \mathrm{b}))\}}\right. \\
\text { se } \log (\mathrm{A} / \mathrm{B})>=\log (\mathrm{a} / \mathrm{b}) \\
\mathrm{f}(\mathrm{A} / \mathrm{B})=\mathrm{fk} \times\left\{[\log (\mathrm{A} / \mathrm{B})-\log (\mathrm{a} / \mathrm{b})] / \sigma_{(\log (\mathrm{a} / \mathrm{b}))}\right\} \mathrm{x} \\
{[\log (\mathrm{a} / \mathrm{b}) / \log (\mathrm{A} / \mathrm{B})]}
\end{gathered}
$$

Quando o elemento "A" for o nutriente Zn:

$$
\text { se } \log (\mathrm{A} / \mathrm{B})<\log (\mathrm{a} / \mathrm{b})
$$

$$
\mathrm{f}(\mathrm{A} / \mathrm{B})=\mathrm{fk} \times\left\{[\log (\mathrm{A} / \mathrm{B})-\log (\mathrm{a} / \mathrm{b})] / \sigma_{(\log (\mathrm{a} / \mathrm{b}))}\right\}
$$$$
\text { se } \log (\mathrm{A} / \mathrm{B})>=\log (\mathrm{a} / \mathrm{b})
$$

$$
\mathrm{f}(\mathrm{A} / \mathrm{B})=\mathrm{fk} x\left\{[\log (\mathrm{A} / \mathrm{B})-\log (\mathrm{a} / \mathrm{b})] / \sigma_{(\log (\mathrm{a} / \mathrm{b}))}\right\} \mathrm{x}
$$$$
[\log (\mathrm{A} / \mathrm{B}) / \log (\mathrm{a} / \mathrm{b})]
$$

Quando o elemento "A" forem os nutrientes Fe, $\mathrm{Mn}$ ou $\mathrm{Cu}$ :

$$
\begin{aligned}
& \text { se } \log (\mathrm{A} / \mathrm{B})<\log (\mathrm{a} / \mathrm{b}) \text { e } \log (\mathrm{A} / \mathrm{B})>\log (\mathrm{a} / \mathrm{b}) / 2 \\
& \mathrm{f}(\mathrm{A} / \mathrm{B})=\mathrm{fkx}\left\{[\log (\mathrm{A} / \mathrm{B})-\log (\mathrm{a} / \mathrm{b})] / \sigma_{(\log (\mathrm{a} / \mathrm{b}))}\right\} \mathrm{x} \\
& {[\log (\mathrm{A} / \mathrm{B}) / \log (\mathrm{a} / \mathrm{b})]} \\
& \text { se } \log (\mathrm{A} / \mathrm{B})<\log (\mathrm{a} / \mathrm{b}) \text { e } \log (\mathrm{A} / \mathrm{B})<=\log \\
& (a / b) / 2 \\
& \mathrm{f}(\mathrm{A} / \mathrm{B})=\mathrm{fk} x\left\{[\log (\mathrm{A} / \mathrm{B})-\log (\mathrm{a} / \mathrm{b})] / \sigma_{(\log (\mathrm{a} / \mathrm{b}))}\right\} \\
& \text { se } \log (\mathrm{A} / \mathrm{B})>=\log (\mathrm{a} / \mathrm{b}) \\
& \mathrm{f}(\mathrm{A} / \mathrm{B})=\mathrm{fk} \times\left\{[\log (\mathrm{A} / \mathrm{B})-\log (\mathrm{a} / \mathrm{b})] / \sigma_{(\log (\mathrm{a} / \mathrm{b}))}\right\}
\end{aligned}
$$

Em cada uma dessas fórmulas, os índices DRIS, para cada nutriente, (In) foram calculados para todas as funções entre dois nutrientes, na forma direta e inversa.

$$
\begin{aligned}
& \mathrm{In}=\left[\mathrm{f}\left(\mathrm{A} / \mathrm{B}_{1}\right)+\mathrm{f}\left(\mathrm{A} / \mathrm{B}_{2}\right)+\ldots+\mathrm{f}\left(\mathrm{A} / \mathrm{B}_{\mathrm{n}}\right)+\ldots . \mathrm{f}\left(\mathrm{B}_{1} / \mathrm{A}\right)\right. \\
& \left.-\mathrm{f}\left(\mathrm{B}_{2} / \mathrm{A}\right)-\ldots-\mathrm{f}\left(\mathrm{B}_{\mathrm{n}} / \mathrm{A}\right)\right] /(2 \mathrm{n}) .
\end{aligned}
$$

Para o cálculo do índice DRIS de matéria seca (Ims), as relações univariadas (teores nutricionais) de cada nutriente foram tomadas em sua expressão inversa:

$$
\operatorname{Ims}=\left[-f(1 / A)-f\left(1 / B_{1}\right)-f\left(1 / B_{2}\right)-\ldots-f\left(1 / B_{n}\right)\right] / n .
$$

em que:

In, índice DRIS do nutriente em questão; Ims, índice de matéria seca do nutriente em questão; $\mathrm{f}(\mathrm{A} / \mathrm{B})$ ou $\mathrm{f}(1 / \mathrm{A})$ ou $\mathrm{f}(1 / \mathrm{B})$ corresponde à função DRIS para dois nutrientes (A e B) quaisquer; $n$ corresponde ao número de nutrientes analisados; $\mathrm{B}_{1}, \mathrm{~B}_{2} \ldots, \mathrm{B}_{\mathrm{n}}$ corresponde aos nutrientes relacionados ao nutriente $\mathrm{A} ; \log (\mathrm{A} / \mathrm{B})$ corresponde à relação entre os nutrientes A e B na amostra; log $(\mathrm{a} / \mathrm{b})$ corresponde à relação entre os nutrientes $\mathrm{A}$ e $\mathrm{B}$ na norma de referência; $\sigma_{(\log (\mathrm{a} / \mathrm{b}))}$ corresponde ao desviopadrão da relação $\mathrm{A}$ e $\mathrm{B}$ na norma de referência; e para a fórmula de Wadt et. al. (2007) o valor fk foi definido como 2 para os nutrientes $\mathrm{N}, \mathrm{P}$ e K, considerados os mais macronutrientes responsivos; como 1,5 para os nutrientes $\mathrm{Zn}$ e Mn, considerados os moderadamente responsivos; igual a 1 para $o$ nutriente $\mathrm{Fe}$, micronutriente pouco responsivo; e igual a 0,5 para os nutrientes $\mathrm{Ca}, \mathrm{Mg}$ e $\mathrm{Cu}$, considerados não responsivos.

Em todos os casos, o índice de balanço nutricional médio foi calculado pela média aritmética do módulo dos índices DRIS.

$\mathrm{Na}$ interpretação dos índices DRIS foram utilizados dois critérios, descritos a seguir.

O primeiro foi o método DRIS Matéria Seca (M-DRIS), que separa cada nutriente em duas categorias, não limitante e limitante (Hallmark et al., 1987), com base no valor do índice DRIS de cada nutriente (In) e na sua comparação com o índice DRIS de matéria seca (Ims):

i) limitante, quando In < 0 e In < Ims; e ii) não limitante, quando In $>0$ ou In $>$ Ims.

O segundo critério foi o método do Potencial de Resposta à Adubação (PRA), o qual classifica o estado nutricional em cinco categorias (Wadt, 2005), com base no valor do índice DRIS e na comparação de seu módulo com o índice de balanço nutricional médio (IBNm):

i) equilibrado, quando $|\mathrm{In}|<\mathrm{IBNm}$; ii) alta probabilidade de insuficiência: $|\mathrm{In}|>\mathrm{IBNm}$, In $<0$ e quando o In foi o índice DRIS de menor valor; iii) 
baixa probabilidade de insuficiência: | In | > IBNm, In $<0$, desde que o In não tenha sido o índice DRIS de menor valor; iv) alta probabilidade de excesso: $|\operatorname{In}|>\operatorname{IBNm}, \mathrm{In}>0$ e quando o In foi o índice DRIS de maior valor; e v) baixa probabilidade de excesso: $|\operatorname{In}|>\operatorname{IBNm}, \operatorname{In}>0$ e desde que o In não tenha sido o índice DRIS de maior valor.

Para comparação dos dois critérios de interpretação dos índices DRIS, inicialmente, computou-se, para cada nutriente, a frequência de pomares em cada um dos possíveis estádios nutricionais, para cada uma das fórmulas de cálculo dos índices DRIS utilizadas.

As frequências obtidas, para cada um dos critérios de interpretação (M-DRIS e PRA), foram comparadas entre si para cada par de fórmulas testadas. Para o critério do M-DRIS, utilizou-se o teste de Qui-Quadrado Exato de Fisher (para tabelas de contingência de duas linhas x duas colunas), e para o critério do PRA, o teste de Qui-Quadrado Corrigido de Yates (para tabelas de contingência de duas linhas $\mathrm{x}$ cinco colunas).

Realizou-se também, para cada fórmula DRIS, a determinação do grau de concordância entre os diagnósticos de cada nutriente obtidos pelo uso do critério do NC, M-DRIS e PRA. Como esses critérios possuem categorias distintas, foram adotadas as seguintes regras:

i) Os diagnósticos foram considerados concordantes quando: a) o nutriente foi considerado limitante pelo método do M-DRIS e insuficiente (alta ou baixa probabilidade) pelo método do PRA; b) o nutriente foi considerado não limitante pelo método do M-DRIS e equilibrado ou em excesso (alta ou baixa probabilidade) pelo método do PRA; c) o nutriente foi deficiente pelo método do $\mathrm{NC}$ e considerado ou limitante pelo método do M-DRIS ou insuficiente (alta ou baixa probabilidade) pelo método do PRA; e d) o nutriente foi suficiente pelo método do NC e considerado ou não limitante pelo método do M-DRIS, ou equilibrado ou em excesso (alta ou baixa probabilidade) pelo método do PRA.

ii) Os diagnósticos foram considerados não concordantes quando: a) o nutriente foi considerado não limitante pelo método do M-DRIS e insuficiente (alta ou baixa probabilidade) pelo método do PRA; b) o nutriente foi considerado limitante pelo método do M-DRIS e equilibrado ou em excesso (alta ou baixa probabilidade) pelo método do PRA; c) o nutriente foi suficiente pelo método do NC e considerado ou limitante pelo método do M-DRIS ou insuficiente (alta ou baixa probabilidade) pelo método do PRA; e d) o nutriente foi deficiente pelo método do NC e considerado, ou não limitante pelo método do M-DRIS ou equilibrado ou em excesso (alta ou baixa probabilidade) pelo método do PRA.
Realizou-se a contagem, para cada nutriente, em função de cada uma das fórmulas DRIS, do número de casos concordantes e discordantes, expressando-se o resultado em percentagem. Para cada um dos critérios de interpretação (M-DRIS e PRA), combinado com as diferentes fórmulas DRIS, e também para o método do $\mathrm{NC}$, foram calculados o número mínimo e máximo, a média e o desviopadrão do número de nutrientes considerados simultaneamente insuficientes, limitantes ou deficientes.

Os cálculos relacionados à obtenção das normas DRIS, dos índices DRIS e dos critérios de interpretação foram feitos por meio do software DRIS Cupuaçu (www.dris.com.br). As contagens e a comparação dos resultados foram feitas em planilha eletrônica (Calc do OpenOffice.org), e as análises estatísticas, pelo software SPSS for Windows 15.0.

\section{RESULTADOS E DISCUSSÃO}

Até o momento não existem, na literatura especializada, valores para a faixa de suficiência ou nível crítico de nutrientes foliares para árvores adultas e em fase de produção para a cultura do cupuaçu. Uma alternativa seria a adoção do método da Chance Matemática para obter as faixas de suficiência, à semelhança do realizado em eucalipto (Wadt et al., 1998b) ou soja (Urano et al., 2007); entretanto, as informações da produtividade das árvores de cupuaçu necessárias para aplicação do método da Chance Matemática foram obtidas por estimativas pouco precisas, o que impossibilita seu uso.

Outra opção seria o uso do próprio método DRIS para obter o nível crítico, por meio de regressão entre os teores foliares e o índice DRIS de cada nutriente, estimando o nível crítico ao se igualar o valor do índice DRIS a zero, ou seja, no ponto de equilíbrio, como feito por Wadt et al. (1998a), em eucaliptos. Contudo, o uso do DRIS para estimar os níveis críticos inviabiliza a utilização deste método (nível crítico) para comparar as fórmulas e os critérios de interpretação dos índices DRIS.

Assim, o nível crítico preliminar (NC) - que correspondeu ao teor foliar do nutriente equivalente a $75 \%$ da amplitude entre os teores de dano fisiológico e o teor ótimo - forneceu um valor independente do DRIS que pôde ser utilizado para comparação dos diagnósticos produzidos pelo sistema DRIS, de forma que os pomares pudessem ser considerados deficientes quando o teor do elemento avaliado fosse menor que o $\mathrm{NC}$ e suficientes quando o teor do nutriente fosse igual ou maior que o $\mathrm{NC}$.

Tomando como base os valores de nível crítico (NC) para os macro e micronutrientes adaptados 
da literatura (Quadro 1), observa-se que a grande maioria dos pomares foi considerada deficiente em P (100 \%), Ca (99 \%) e Zn (98 \%) (Quadro 2). Os teores de Fe e $\mathrm{K}$ foram também apontados como limitantes em 75 e $73 \%$ dos pomares avaliados, respectivamente, enquanto o $\mathrm{N}$ foi apontado como deficiente em $65 \%$ destes (Quadro 2). Esses resultados podem ser decorrentes do fato de que parte dos padrões utilizados foi adaptada de valores obtidos em folhas de mudas de cupuaçueiros, mesmo utilizando-se teores de folhas maduras (Salvador et al., 1994).

Os diagnósticos produzidos pelas diferentes fórmulas e critérios de interpretação dos índices DRIS não foram, em geral, consistentes com os resultados obtidos pelo $\mathrm{NC}$ quanto aos nutrientes considerados deficientes. O critério M-DRIS, quando aplicado nas fórmulas de Jones e de Beaufils, não destacou nenhum nutriente como de maior relevância - considerada essa relevância com base na frequência, como limitante, nos pomares avaliados.

Pela fórmula de Jones, Fe foi apontado como o mais limitante em $59 \%$ dos pomares, seguido pelo $\mathrm{P}(52 \%)$ e Mn (50 \%); pela fórmula de Beaufils, a frequência com que $\mathrm{Fe}$ foi apontado como mais limitante foi de $59 \%$, seguido pelo $\mathrm{Mn}(47 \%)$ e por N, P e Zn, todos com $47 \%$ de pomares apresentando deficiência (Quadro 3).

Por outro lado, usando a fórmula de Wadt et al., foram considerados deficientes o Zn e o Mn (100\% dos pomares) e o N (99\%), seguido pelo Fe (85\%). $\mathrm{Ca}, \mathrm{Cu}$ e $\mathrm{P}$ foram deficientes em no máximo $1 \%$ dos pomares (Quadro 3).

Comparando as diferentes fórmulas DRIS pelo teste de Qui-quadrado, observa-se que as fórmulas de Jones e Beaufils foram semelhantes entre si

Quadro 2. Percentagem de pomares classificados pelo método do nível crítico preliminar, para cada nutriente, como deficiente ou suficiente

\begin{tabular}{ccc}
\hline Nutriente & Deficiente & Suficiente \\
\hline $\mathrm{N}$ & $65 \%$ & $35 \%$ \\
$\mathrm{P}$ & $100 \%$ & $0 \%$ \\
$\mathrm{~K}$ & $73 \%$ & $27 \%$ \\
$\mathrm{Ca}$ & $99 \%$ & $1 \%$ \\
$\mathrm{Mg}$ & $54 \%$ & $46 \%$ \\
$\mathrm{Zn}$ & $98 \%$ & $2 \%$ \\
$\mathrm{Fe}$ & $75 \%$ & $25 \%$ \\
$\mathrm{Mn}$ & $6 \%$ & $94 \%$ \\
$\mathrm{Cu}$ & $10 \%$ & $90 \%$ \\
\hline
\end{tabular}

$(\mathrm{p}<0,05)$, exceto para $\mathrm{K}$ e $\mathrm{Mg}$, ao passo que a fórmula de Wadt et al. apresentou distribuição de frequência diferente das demais fórmulas para todos os nutrientes testados (Quadro 3).

Foi notória a tendência de que o critério do M-DRIS, com as fórmulas de Jones e Beaufils, resultou em proporção de pomares deficientes, na maioria dos casos, entre 35 e $65 \%$, ou seja, mais ou menos $15 \%$ em torno do valor mais provável para a casualidade (50 \%).

A utilização do critério do Potencial de Resposta à Adubação com as fórmulas de Jones e de Beaufils alterou pouco a importância relativa dos nutrientes considerados insuficientes na maior parte dos pomares: o Fe continuou sendo o nutriente com maior frequência de insuficiência (49 a $51 \%$, pelos métodos de Jones e Beaufils, respectivamente), seguido pelo Mn (35 e $32 \%$, respectivamente para os métodos de Jones e Beaufils) e K (22 e $27 \%$, respectivamente para os métodos de Beaufils e Jones). Nesses casos, entretanto, a frequência da distribuição do número de pomares em cada classe de estado nutricional não diferiu entre as duas fórmulas, para qualquer um dos nutrientes testados (Quadro 4). Ambas as fórmulas (Jones e Beaufils) tenderam a indicar alto número de nutrientes nutricionalmente equilibrados.

Por outro lado, o uso da fórmula de Wadt et al., associada ao critério do Potencial de Resposta à Adubação, detectou a importância com que os nutrientes foram considerados mais deficientes: $\mathrm{N}$ foi considerado o nutriente com maior ocorrência de insuficiência com alta probabilidade, em $75 \%$ dos pomares, seguido pelo $\mathrm{Mn}$, em $25 \%$ dos pomares; com baixa probabilidade de insuficiência foram apontados o N (24\% dos pomares), seguido do $\mathrm{Mn}$ $(74 \%)$ e do $\mathrm{Zn}(82 \%)$. Todos os demais nutrientes foram considerados equilibrados $(\mathrm{K}, \mathrm{Ca}, \mathrm{Mg}, \mathrm{Fe}$ e $\mathrm{Cu}$ ) ou em excesso (P) (Quadro 4).

A elevada frequência de pomares com deficiência em $\mathrm{N}, \mathrm{Ca}$ e $\mathrm{Fe}$, indicadas pelo método do $\mathrm{NC}$, não tem suporte na literatura (Ayres \& Alfaia, 1998; Alfaia \& Ayres, 2004, 2007), apesar de haver poucos estudos conclusivos, pela escassez de trabalhos de adubação com essa cultura.

Respostas a adubações de $\mathrm{P}$ e $\mathrm{Ca}$, sem o acompanhamento da aplicação simultânea de $\mathrm{N}$ e $\mathrm{K}$, não têm sido relatadas na literatura. Embora a omissão de $\mathrm{P}$ represente perda de produtividade para o cupuaçueiro (Alfaia \& Ayres, 2004), seu efeito benéfico depende da aplicação simultânea de outros nutrientes, uma vez que, sem a adição de $\mathrm{N}$ e $\mathrm{K}$, a demanda da planta por $\mathrm{P}$ pode ser menor e suprida satisfatoriamente pela associação micorrízica, a qual, inclusive, parece não ser afetada pela disponibilidade desse nutriente $(\mathrm{P})$ no solo. Trabalho independente conduzido por Silva Júnior 
Quadro 3. Percentagem de pomares classificados pelo método do DRIS Matéria Seca, para cada nutriente e em função das diferentes fórmulas DRIS, como limitante ou não limitante; valor da significância para o teste de Qui-Quadrado $(\chi 2)$ na comparação das frequências absolutas (contagens) para as classificações obtidas entre as fórmulas DRIS ( $\mathrm{J}=$ Jones, $\mathrm{B}=$ Beaufils, $\mathrm{W}=$ Wadt et al), tomadas duas a duas

\begin{tabular}{|c|c|c|c|}
\hline Fórmulas DRIS & Limitante & \multicolumn{2}{|c|}{ Não Limitante } \\
\hline & & $\mathrm{N}$ & \\
\hline Jones & $48 \%$ & & $52 \%$ \\
\hline Beaufils & $41 \%$ & & $59 \%$ \\
\hline Wadt et al. & $99 \%$ & & $1 \%$ \\
\hline$\chi^{2}$ & $\mathrm{~J} \times \mathrm{B}: 0,30$ & $\begin{array}{c}\mathrm{J} \times \mathrm{W}: 0,00 \\
\mathrm{P}\end{array}$ & B x W: 0,00 \\
\hline Jones & $52 \%$ & & $48 \%$ \\
\hline Beaufils & $41 \%$ & & $59 \%$ \\
\hline Wadt et al. & $1 \%$ & & $99 \%$ \\
\hline$\chi^{2}$ & $\mathrm{~J} \times \mathrm{B}: 0,07$ & $\begin{array}{c}\mathrm{J} \times \mathrm{W}: 0,00 \\
\mathrm{~K}\end{array}$ & B x W: 0,00 \\
\hline Jones & $48 \%$ & & $52 \%$ \\
\hline Beaufils & $35 \%$ & & $65 \%$ \\
\hline Wadt et al. & $22 \%$ & & $78 \%$ \\
\hline$\chi^{2}$ & $\mathrm{~J}$ x B: 0,04 & $\begin{array}{c}\mathrm{J} \times \mathrm{W}: 0,00 \\
\mathrm{Ca}\end{array}$ & $\mathrm{B} \times \mathrm{W}: 0,00$ \\
\hline Jones & $41 \%$ & & $59 \%$ \\
\hline Beaufils & $38 \%$ & & $62 \%$ \\
\hline Wadt et al. & $12 \%$ & & $88 \%$ \\
\hline$\chi^{2}$ & $\mathrm{~J}$ x B: 0,64 & $\begin{array}{c}\mathrm{J} \times \mathrm{W}: 0,00 \\
\mathrm{Mg}\end{array}$ & B x W: 0,00 \\
\hline Jones & $38 \%$ & & $62 \%$ \\
\hline Beaufils & $25 \%$ & & $75 \%$ \\
\hline Wadt et al. & $0 \%$ & & $100 \%$ \\
\hline$\chi^{2}$ & $\mathrm{~J}$ x B: 0,02 & $\begin{array}{c}\mathrm{J} \times \mathrm{W}: 0,00 \\
\mathrm{Zn}\end{array}$ & $\mathrm{B} \times \mathrm{W}: 0,00$ \\
\hline Jones & $44 \%$ & & $56 \%$ \\
\hline Beaufils & $41 \%$ & & $59 \%$ \\
\hline Wadt et al. & $100 \%$ & & $0 \%$ \\
\hline$\chi^{2}$ & $\mathrm{~J} \times \mathrm{B}: 0,64$ & $\begin{array}{c}\mathrm{J} \times \mathrm{W}: 0,00 \\
\mathrm{Fe}\end{array}$ & B x W: 0,00 \\
\hline Jones & $59 \%$ & & $41 \%$ \\
\hline Beaufils & $59 \%$ & & $41 \%$ \\
\hline Wadt et al. & $85 \%$ & & $15 \%$ \\
\hline$\chi^{2}$ & $\mathrm{~J} \times \mathrm{B}: 1.00$ & $\begin{array}{c}\mathrm{J} \times \mathrm{W}: 0,00 \\
\mathrm{Mn}\end{array}$ & B x W: 0,00 \\
\hline Jones & $50 \%$ & & $50 \%$ \\
\hline Beaufils & $47 \%$ & & $53 \%$ \\
\hline Wadt et al. & $100 \%$ & & $0 \%$ \\
\hline$\chi^{2}$ & $\mathrm{~J} x \mathrm{~B}: 0,73$ & $\begin{array}{c}\mathrm{J} \times \mathrm{W}: 0,00 \\
\mathrm{Cu}\end{array}$ & $\mathrm{B} \times \mathrm{W}: 0,00$ \\
\hline Jones & $39 \%$ & & $61 \%$ \\
\hline Beaufils & $33 \%$ & & $67 \%$ \\
\hline Wadt et al. & $0 \%$ & & $100 \%$ \\
\hline$\chi^{2}$ & $\mathrm{~J} \times \mathrm{B}: 0,28$ & $\mathrm{~J} \times \mathrm{W}: 0,00$ & B x W: 0,00 \\
\hline
\end{tabular}

\& Cardoso (2006) indica que a associação micorrízica no cupuaçueiro é mais intensa no período úmido, justamente aquele de maior crescimento das plantas.

Por sua vez, a indicação de excesso de $\mathrm{P}$, com teores foliares sempre inferiores a $1 \mathrm{~g} \mathrm{~kg}^{-1} \mathrm{de}$ $\mathrm{P}$, parece também improvável, ainda mais se considerado que a indicação de excesso estaria sugerindo queda na produtividade com a aplicação desse nutriente.

Quanto ao Ca, o efeito de sua aplicação parece estar associado à melhoria das condições químicas do solo em função de sua aplicação na forma de calagem, diminuindo a acidez do solo e, assim, favorecendo a disponibilidade dos nutrientes, e não por redução de sua deficiência na plantas (Alfaia et al., 2007). Respostas à adição de $\mathrm{Zn}$ têm sido relatadas em mudas de cupuaçueiros (Fernandes et al., 2003), embora com forte antagonismo, com a aplicação conjunta de P. Assim, as deficiências indicadas pelo método do NC não parecem ser prováveis na proporção em que são sugeridas. Tampouco seria esperada, como indicado pelo método DRIS com as fórmulas de Jones ou Beaufils, uma frequência de pomares deficientes para a maioria dos nutrientes, entre 35 e $65 \%$.

A deficiência de $\mathrm{N}$, apontada pelos métodos do NC e DRIS, usando-se a fórmula de Wadt et al. também não tem respaldo na literatura; os poucos ensaios de adubação não têm mostrado a deficiência desse nutriente nos pomares de cupuaçueiros quando aplicado isoladamente, e sim apenas em aplicações acompanhadas de P e K (Alfaia et al., 2004). Resultados preliminares apontam o N como o único nutriente reconhecidamente não responsivo à adubação por essa cultura (Yuyama et al., 2008).

Avaliando o grau de concordância entre o método DRIS e o NC, observa-se elevado grau de discordância para a maioria dos nutrientes (sempre acima de $50 \%$ ), exceto para $\mathrm{Fe}$, quando se utiliza a fórmula de Jones ou de Beaufils com o método do Potencial de Resposta à Adubação, sendo relativamente alta essa discordância (acima de $37 \%$ ) quando se adota o critério do M-DRIS (Quadro 5).

Utilizando a fórmula de Wadt e colaboradores, a concordância com o método do NC foi alta para N, Zn, $\mathrm{Fe}$ e $\mathrm{Cu}$ (acima de $73 \%$ ) e muito baixa para P, Ca e Mn (abaixo de $13 \%$ ). A maior divergência foi que o DRIS (fórmula de Wadt et al. e critério do PRA) não indicou insuficiência para $\mathrm{P}$ e Ca, enquanto o $\mathrm{NC}$ o fez; além disso, para $\mathrm{Mn}$, o DRIS indicou insuficiência quando o NC não a indicou (Quadros 2 e 4).

Embora a distribuição de frequência de diagnósticos semelhantes tenha sido similar $(p<0,05)$ entre as fórmulas de Jones e Beaufils para a maioria dos nutrientes, o grau de concordância dos diagnósticos por essas duas fórmulas foi alterado pelo critério de interpretação, variando de $68 \%$ (para $\mathrm{N}$, com a fórmula de Jones) a $93 \%$ (para $\mathrm{Cu}$ ). 
Quadro 4. Percentagem de pomares classificados pelo método do Potencial de Resposta à Adubação, para cada nutriente e em função das diferentes fórmulas DRIS, como insuficientes com alta probabilidade (IAP), insuficientes com baixa probabilidade (IBP), equilibrados (EQ), excesso com baixa probabilidade (EXBP) e excesso com alta probabilidade (EXAP); valor da significância para o teste de Qui-Quadrado ( $\chi 2)$ na comparação das frequências absolutas (contagens) para as classificações obtidas entre as fórmulas DRIS (J = Jones, $\mathrm{B}=$ Beaufils, $\mathrm{W}=$ Wadt et al.), tomadas duas a duas

Fórmulas DRIS IAP IBP EQ EXBP EXAP

\begin{tabular}{|c|c|c|c|}
\hline \multirow[b]{2}{*}{ Jones } & \multicolumn{3}{|l|}{$\mathrm{N}$} \\
\hline & $12 \% \quad 73 \%$ & $8 \%$ & $3 \%$ \\
\hline Beaufils & $10 \% 74 \%$ & $10 \%$ & $5 \%$ \\
\hline \multirow{2}{*}{$\begin{array}{l}\text { Wadt et al. } \\
\chi 2\end{array}$} & $24 \%$ & $0 \%$ & $1 \%$ \\
\hline & $\begin{array}{r}\mathrm{J} \times \mathrm{B}: 0,75 \mathrm{~J} \times \mathrm{W}: 0,00 \\
\mathrm{P}\end{array}$ & \multicolumn{2}{|c|}{ B x W: 0,00} \\
\hline Jones & $13 \% 61 \%$ & $11 \%$ & $7 \%$ \\
\hline Beaufils & $14 \% 59 \%$ & $12 \%$ & $7 \%$ \\
\hline Wad & $1 \%$ & $1 \%$ & $98 \%$ \\
\hline$\chi^{2}$ & \multicolumn{3}{|c|}{$\begin{array}{c}\mathrm{J} \times \mathrm{B}: 0,98 \mathrm{~J} \times \mathrm{K} \\
\mathrm{W}\end{array}$} \\
\hline Jones & $20 \% \quad 46 \%$ & $14 \%$ & $13 \%$ \\
\hline Bea & $17 \% \quad 52 \%$ & $14 \%$ & $12 \%$ \\
\hline Wad & $1 \% 97 \%$ & $1 \%$ & $1 \%$ \\
\hline$\chi^{2}$ & \multicolumn{3}{|c|}{$\begin{array}{c}J \times \mathrm{B}: 0,84 \mathrm{~J} \times \mathrm{W}: 0,00 \mathrm{~B} \times \mathrm{W}: 0,00 \\
\mathrm{Ca}\end{array}$} \\
\hline Jon & $12 \% \quad 50 \%$ & $20 \%$ & $8 \%$ \\
\hline Bea & $12 \% \quad 57 \%$ & $15 \%$ & $8 \%$ \\
\hline Wadt et al. & $0 \% 100 \%$ & $0 \%$ & $0 \%$ \\
\hline$\chi^{2}$ & \multicolumn{3}{|c|}{$\begin{array}{c}\mathrm{J} \times \mathrm{B}: 0,63 \mathrm{~J} \times \mathrm{W}: 0,00 \mathrm{~B} \times \mathrm{W}: 0,00 \\
\mathrm{Mg}\end{array}$} \\
\hline Jon & $8 \% \quad 63 \%$ & $15 \%$ & $8 \%$ \\
\hline Bea & $7 \% \quad 58 \%$ & $20 \%$ & $10 \%$ \\
\hline Wadt et al. & $0 \% 100 \%$ & $0 \%$ & $0 \%$ \\
\hline$\chi^{2}$ & \multicolumn{3}{|c|}{$\begin{array}{c}\mathrm{J} \times \mathrm{B}: 0,72 \mathrm{~J} \times \mathrm{W}: 0,00 \mathrm{~B} \times \mathrm{W}: 0,00 \\
\mathrm{Zn}\end{array}$} \\
\hline Jon & $17 \% \quad 58 \%$ & $10 \%$ & $8 \%$ \\
\hline Bea & $15 \% \quad 59 \%$ & $9 \%$ & $7 \%$ \\
\hline Wadt et al. & $82 \% \quad 18 \%$ & $0 \%$ & $0 \%$ \\
\hline$\chi^{2}$ & \multicolumn{3}{|c|}{$\begin{array}{c}\mathrm{J} \times \mathrm{B}: 0,91 \mathrm{~J} \times \mathrm{W}: 0,00 \mathrm{~B} \times \mathrm{W}: 0,00 \\
\mathrm{Fe}\end{array}$} \\
\hline Jon & $28 \% \quad 25 \%$ & $8 \%$ & $18 \%$ \\
\hline Beal & $20 \% \quad 25 \%$ & $7 \%$ & $18 \%$ \\
\hline Wadt et al. & $0 \% 100 \%$ & $0 \%$ & $0 \%$ \\
\hline$\chi^{2}$ & \multicolumn{3}{|c|}{$\begin{array}{c}\mathrm{J} \times \mathrm{B}: 0,26 \mathrm{~J} \times \mathrm{W}: 0,00 \mathrm{~B} \times \mathrm{W}: 0,00 \\
\mathrm{Mn}\end{array}$} \\
\hline Jon & $17 \% \quad 29 \%$ & $12 \%$ & $24 \%$ \\
\hline Beaufils & $14 \% \quad 29 \%$ & $14 \%$ & $24 \%$ \\
\hline Wadt et al. & $74 \%$ & $0 \%$ & $0 \%$ \\
\hline$\chi^{2}$ & \multicolumn{3}{|c|}{$\begin{array}{c}\mathrm{J} \times \mathrm{B}: 0,94 \mathrm{~J} \times \mathrm{Cu}: 0,00 \mathrm{~B} \times \mathrm{W}: 0,00 \\
\mathrm{Cu}\end{array}$} \\
\hline Jones & $8 \% \quad 44 \%$ & $19 \%$ & $10 \%$ \\
\hline $\mathrm{Bec}$ & $12 \% \quad 50 \%$ & $14 \%$ & $10 \%$ \\
\hline Wac & $0 \% 100 \%$ & $0 \%$ & $0 \%$ \\
\hline$\chi^{2}$ & \multicolumn{3}{|c|}{$J \times B: 0,35 \mathrm{~J} \times \mathrm{W}: 0,00 \mathrm{~B} \times \mathrm{W}: 0,00$} \\
\hline
\end{tabular}

Em geral, a fórmula de Wadt et al. resultou em diagnósticos mais consistentes entre si, independentemente do critério de interpretação adotado; o grau de concordância foi igual ou acima de 99 \% para N, P, Mg, Mn e Cu (Quadro 5). Destaca-se, entretanto, a grande discordância no diagnóstico do $\mathrm{Fe}$, com apenas $15 \%$ de diagnósticos concordantes (Quadro 5), uma vez que o M-DRIS indicou esse nutriente como limitante em $85 \%$ dos pomares e o PRA não indicou insuficiência de $\mathrm{Fe}$ em nenhum deles.

Quadro 5. Percentagem de diagnósticos concordantes $(\mathrm{C}(=))$ e discordantes $(\mathrm{D}(<>))$, entre os métodos do nível crítico (NC), DRIS de Matéria Seca (M-DRIS) e Potencial de Resposta à Adubação (PRA), combinados com as fórmulas de Jones, Beaufils ou Wadt et al., para um total de 153 pomares de cupuaçueiros

\begin{tabular}{|c|c|c|c|}
\hline Fórmulas & $\begin{array}{c}\text { NC X } \\
\text { M-DRIS }\end{array}$ & NC X PRA & $\begin{array}{l}\text { M-DRIS X } \\
\text { PRA }\end{array}$ \\
\hline & $\overline{\mathrm{D}(<>) \quad \mathrm{C}(=)}$ & $\mathrm{D}(<>) \quad \mathrm{C}(=)$ & $\overline{\mathrm{D}(<>) \quad \mathrm{C}(=)}$ \\
\hline
\end{tabular}

\begin{tabular}{|c|c|c|c|c|c|c|}
\hline \multirow[b]{2}{*}{ Jones } & \multicolumn{6}{|c|}{$\mathrm{N}$} \\
\hline & $49 \%$ & $51 \%$ & $80 \%$ & $20 \%$ & $32 \%$ & 68 \\
\hline Beaufils & $50 \%$ & $50 \%$ & $81 \%$ & $19 \%$ & $29 \%$ & 71 \\
\hline \multirow[t]{2}{*}{ Wadt et al. } & $34 \%$ & $66 \%$ & $35 \%$ & $65 \%$ & $1 \%$ & م \\
\hline & \multicolumn{6}{|c|}{$\mathrm{P}$} \\
\hline Jones & $48 \%$ & $52 \%$ & $79 \%$ & $21 \%$ & $31 \%$ & 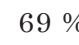 \\
\hline Beaufils & $59 \%$ & $41 \%$ & $78 \%$ & $22 \%$ & $22 \%$ & 78 \\
\hline \multirow[t]{2}{*}{ Wadt et al. } & $99 \%$ & $1 \%$ & $99 \%$ & $1 \%$ & $1 \%$ & 99 \\
\hline & \multicolumn{6}{|c|}{ K } \\
\hline Jones & $55 \%$ & $45 \%$ & $50 \%$ & $50 \%$ & $21 \%$ & 10 \\
\hline Beaufils & $58 \%$ & $42 \%$ & $54 \%$ & $46 \%$ & $14 \%$ & 86 \\
\hline \multirow[t]{2}{*}{ Wadt et al. } & $61 \%$ & $39 \%$ & $98 \%$ & $2 \%$ & $22 \%$ & 78 \\
\hline & \multicolumn{6}{|c|}{$\mathrm{Ca}$} \\
\hline Jones & $60 \%$ & $40 \%$ & $77 \%$ & $23 \%$ & $20 \%$ & 00 \\
\hline Beaufils & $63 \%$ & $37 \%$ & $79 \%$ & $21 \%$ & $18 \%$ & 82 \\
\hline \multirow[t]{2}{*}{ Wadt et al. } & $87 \%$ & $13 \%$ & $100 \%$ & $0 \%$ & $12 \%$ & \\
\hline & \multicolumn{6}{|c|}{$\mathrm{Mg}$} \\
\hline Jones & $56 \%$ & $44 \%$ & $69 \%$ & $31 \%$ & $24 \%$ & \\
\hline Beaufils & $54 \%$ & $46 \%$ & $65 \%$ & $35 \%$ & $14 \%$ & \\
\hline Wadt et al. & $54 \%$ & $46 \%$ & $100 \%$ & $0 \%$ & $0 \%$ & 10 \\
\hline
\end{tabular}

\section{$\mathrm{Zn}$}

$\begin{array}{lllllll}\text { Jones } & 54 \% & 46 \% & 73 \% & 27 \% & 19 \% & 81 \%\end{array}$

Beaufils $\quad 59 \% \quad 41 \% \quad 73 \% \quad 27 \% \quad 18 \% \quad 82 \%$

Wadt et al. $\quad 2 \% \quad 98 \% \quad 20 \% \quad 80 \% \quad 18 \% \quad 82 \%$

$\mathrm{Fe}$

Jones $\quad 41 \% \quad 59 \% \quad 27 \% \quad 73 \% \quad 10 \% \quad 90 \%$

Beaufils $\quad 41 \% \quad 59 \% \quad 28 \% \quad 72 \% \quad 9 \% \quad 91 \%$

Wadt et al. $\quad 27 \% \quad 73 \% \quad 100 \% \quad 0 \% \quad 85 \% \quad 15 \%$

$\mathrm{Mn}$

$\begin{array}{lllllllll}\text { Jones } & 52 \% & 48 \% & 58 \% & 42 \% & 14 \% & 86 \%\end{array}$

Beaufils $\quad 49 \% \quad 51 \% \quad 56 \% \quad 44 \% \quad 14 \% \quad 86 \%$

Wadt et al. $94 \% \quad 6 \% \quad 94 \% \quad 6 \% \quad 1 \% \quad 99 \%$

$\mathrm{Cu}$

Jones $\quad 41 \% \quad 59 \% \quad 60 \% \quad 40 \% \quad 13 \% \quad 87 \%$

Beaufils $\quad 37 \% \quad 63 \% \quad 65 \% \quad 35 \% \quad 7 \% \quad 93 \%$

Wadt et al. $\quad 10 \% \quad 90 \% \quad 100 \% \quad 0 \% \quad 0 \% \quad 100 \%$ 
Comparando os dois critérios de interpretação dos índices DRIS com base apenas na frequência com que os nutrientes foram classificados nos diferentes grupos de estado nutricional, Partelli et al. (2006) concluíram não haver diferença entre os dois critérios; contudo, como não foi avaliado o grau de concordância para os diagnósticos, individualmente para cada pomar e nutriente, poderia haver diferença no grau de concordância, mesmo as frequências sendo iguais $(p<0,05)$, conforme demonstrado neste trabalho.

Na utilização das fórmulas de Jones ou de Beaufils foi encontrada superestimativa da deficiência de alguns nutrientes, como $\mathrm{Mn}$, Mo e $\mathrm{Zn}$, em arroz irrigado, mesmo em solos bem providos desses nutrientes (Guindani et al., 2009).

A quantidade de nutrientes apontada como deficiente, limitante e insuficiente, respectivamente pelos critérios do NC, M-DRIS e PRA, foi de 2 a 8 , para o método do $\mathrm{NC}$, com média de seis nutrientes deficientes por pomar (Quadro 6). Ao se usar o critério do M-DRIS, o número de nutrientes considerados limitantes variou de 0 a 7 , sendo, entretanto, dependente da fórmula utilizada: para a fórmula de Beaufils esse número variou de 0 a 7 , enquanto para a de Wadt e colaboradores ele variou de 3 a 6 (Quadro 6). Quanto ao critério do Potencial de Resposta à Adubação, a variação para as três fórmulas de cálculo foi de 1 a 4; o valor mais provável foi de dois (fórmulas de Jones ou Beaufils) ou três nutrientes (fórmula de Wadt e colaboradores) insuficientes por pomar. Também, com o critério do Potencial de Resposta à Adubação foram obtidos os menores valores para o desvio-padrão do número de nutrientes considerados insuficientes.

Os métodos DRIS e NC podem estar apresentando muitos casos de diagnósticos falso-positivos (Beverly, 1993), ou seja, indicação de insuficiência quando esta não existe realmente. Essa indicação poderá resultar em diminuição da lucratividade dos pomares, por demandar adubações acima da necessidade dos cupuaçueiros, além dos impactos ambientais negativos, decorrentes do uso excessivo e desnecessário de fertilizantes para suprir as deficiências apontadas. O uso do Potencial de Resposta à Adubação, com as fórmulas de Jones e Beaufils, também pode estar resultando em diagnósticos falso-negativos para a grande maioria dos nutrientes (grande proporção de situações de equilíbrio nutricional quando haveria deficiências verdadeiras). Quando se adota a fórmula de Wadt et al., também se observa a possibilidade de diagnósticos falso-negativos, como para $\mathrm{P}$.

Os resultados indicam que nenhum dos métodos utilizados mostrou-se completamente compatível com os demais, sendo necessários novos estudos para que se possa determinar qual o mais apropriado. A fórmula de Wadt e colaboradores mostrou menor dependência do critério de interpretação dos índices DRIS (M-DRIS ou PRA) que as fórmulas de Jones e de Beaufils.

Para $\mathrm{N}$ e $\mathrm{P}$, os resultados obtidos pela fórmula de Wadt et al. foram, com base nos resultados da literatura, inferiores aos obtidos pelas fórmulas de Jones ou de Beaufils. Quanto ao uso da fórmula de Wadt e colaboradores, há necessidade de ajustes nos coeficientes de sensibilidade, conforme proposto por Maia (1999) e Wadt et al. (2007), para que sejam minimizadas as possibilidades de diagnósticos falso-negativos, que resultariam em não aplicação do nutriente quando isso se fizer necessário.

\section{CONCLUSÕES}

1. O nível crítico preliminar adotado neste trabalho não foi adequado na avaliação do estado nutricional de pomares de cupuaçu. No mesmo

Quadro 6. Mínimo, máximo, média e desvio-padrão amostral para a quantidade de nutrientes indicados como deficientes, limitantes e insuficientes, respectivamente, para os métodos do Nível Crítico (NC), DRIS de matéria seca (M-DRIS) e do Potencial de Resposta à Adubação (PRA), utilizando-se as fórmulas de Jones, Beaufils e Wadt et al., para os métodos do M-DRIS e PRA. Estatísticas obtidas da avaliação nutricional de 153 pomares de cupuaçueiros

\begin{tabular}{|c|c|c|c|c|c|}
\hline Método & Fórmula DRIS & Mínimo & Máximo & Média & Desvio-Padrão \\
\hline $\mathrm{NC}$ & não se aplica & 2 & 8 & 5,8 & 1,1 \\
\hline M-DRIS & Jones & 1 & 7 & 4,2 & 1,2 \\
\hline M-DRIS & Beaufils & 0 & 7 & 3,6 & 1,3 \\
\hline M-DRIS & Wadt et al & 3 & 6 & 4,2 & 0,7 \\
\hline PRA & Jones & 1 & 4 & 2,3 & 0,7 \\
\hline PRA & Beaufils & 1 & 4 & 2,2 & 0,7 \\
\hline PRA & Wadt et al & 1 & 4 & 2,8 & 0,4 \\
\hline
\end{tabular}


sentido, nenhuma fórmula DRIS ou critério de interpretação dos índices mostrou-se mais indicado que os demais para ser utilizado na avaliação do estado nutricional dos pomares.

2. Na avaliação do estado nutricional dos pomares, as fórmulas de Beaufils (1973) e de Jones (1981) foram semelhantes entre si, porém distintas da fórmula de Wadt et al. (2007).

3. Os diagnósticos produzidos pelos diferentes critérios de interpretação dos índices DRIS (Potencial de Resposta à Adubação ou método do M-DRIS) não foram consistentes entre si quando se adotou a fórmula de Beaufils (1973) ou a de Jones (1981). A exceção foi a fórmula de Wadt et al. (2007), que mostrou maior consistência entre os diagnósticos, independentemente do critério utilizado para sua interpretação.

\section{AGRADECIMENTOS}

Ao CNPq e à FUNTAC, pelo apoio financeiro ao projeto de pesquisa; e aos produtores e técnicos do Projeto de Reflorestamento Econômico Consorciado Adensado (RECA), de Vila Califórnia, Porto Velho, RO, pelo apoio logístico e pela colaboração nos trabalhos de campo.

\section{LITERATURA CITADA}

ALFAIA, S.S. \& AYRES, M.I.C. Efeito de doses de nitrogênio, fósforo e potássio em duas cultivares de cupuaçu, com e sem semente, na região da Amazônia Central. R. Bras. Frutic., 26, p. 320-325, 2004.

ALFAIA, S.S.; RIBEIRO, G.A.; NOBRE, A.D.; LUIZÃO, R.C. \& LUIZÃO, F.J. Evaluation of soil fertility in smallholder agroforestry systems and pastures in western Amazonia. Agric., Ecosyst. Environ., 102:409-414, 2004.

AYRES, M.I.C. \& ALFAIA, S.S. Calagem e adubação potássica na produção do cupuaçuzeiro em sistemas agroflorestais da Amazônia Ocidental. Pesq. Agropec. Bras., 42:957-963, 2007.

AYRES, M.I.C. \& ALFAIA, S.S. Efeito de NPK, calagem e micronutrientes na produção de frutos do cupuaçuzeiro. R. Bras. Frutic., 32:863-870, 1998.

BEAUFILS, E.R. Diagnosis and Recommendation Integrated System (DRIS). Pietermaritzburg, University of Natal, 1973. 132p. (Soil Scince Bulletin, 1)

BEVERLY, R.B. Comparison of DRIS and alternative nutrient diagnostic methods for soybean. J. Plant Nutr., 10:901920, 1987.

BEVERLY, R.B. DRIS Diagnoses of soybean nitrogen, phosphorus, and potassium status are unsatisfactory. J. Plant Nutr., 16:1431-1447, 1993.
CARMO, C.A.F.S.; ARAÚJO, W.S.; BERNARDI, A.C.C. \& SALDANHA, M.F.C. Métodos de análise de tecidos vegetais utilizados pela Embrapa Solos. Rio de Janeiro, Embrapa Solos, 2000. 41p.

COSTA, E.L. Exportação de nutrientes em frutos de cupuaçu (Theobroma grandiflorum) em três solos da Amazônia Central. Manaus, Universidade Federal do Amazonas, 2006. 39p. (Tese de Mestrado)

DIAS, J.R.M.; WADT, P.G.S.; LEMOS, C.O.; DELARMELINDA, E.A.; TAVELLA, L.B. \& SOLINO, J.S. Uso de normas DRIS log-transformadas para a cultura do cupuaçu. Acta Amaz., 39:596-601, 2009.

FERNANDES, A.R.; CARVALHO, J.G. \& MELO, P.C. Efeito do fósforo e do zinco sobre o crescimento de mudas do cupuaçuzeiro (Theobroma grandiflorum schum.). R. Cerne, 9:221-230, 2003.

GUINDANI, R.R.H.P.; ANGHINONI, I. \& NACHTIGALL, G.R. DRIS na avaliação do estado nutricional do arroz irrigado por inundação. R. Bras. Ci. Solo, 33:109-118, 2009.

HALLMARK, W.B.; WALWORTH, J.L.; SUMNER, M.E.; MOOY, C.J.; PESEK, J. \& SHAO, K.P. Separating limiting from non-limiting nutrients. J. Plant Nutr., 10:1381-1390, 1987.

JONES, W.W. Proposed modifications of the diagnosis and recommendation integrated system (DRIS) for interpreting plant analyses. Comm. Soil Sci. Plant Anal., 12:785-794, 1981.

LOPES, C.M.D.A. \& SILVA, N.M. Impacto econômico da broca do cupuaçu, Conotrachelus humeropictus field (Coleoptera: Curculionidae) nos estados do Amazonas e Rondônia. Soc. Entomol. Brasil, 27:45-49, 1998.

MAIA, C.E. Análise crítica da fórmula original de Beaufils no cálculo dos índices DRIS: A constante de sensibilidade. In: WADT, P.G.S. \& MALAVOLTA, E., ed. Monitoramento nutricional para a recomendação de adubação para as culturas. Piracicaba, POTAFÓs, 1999. 17p. CD ROOM.

MOURÃO, F.A.A.; AZEVEDO, J.C. \& NICK, J.A. Função e ordens da razão dos nutrientes no estabelecimento de normas DRIS em laranja "Valência!". Pesq. Agropec. Bras., 37:185-192, 2002.

PARTELLI, F.L.; VIEIRA, H.D.; MONNERAT, P.H. \& VIANA, A.P. Estabelecimento de normas DRIS em cafeeiro conilon orgânico e convencional no Estado do Espírito Santo. R. Bras. Ci. Solo, 30:20-25, 2006.

SALVADOR, J.O.; MURAOKA, T.; ROSSETO, R. \& IBEIRO, G.A. Sintomas de deficiências nutricionais em cupuaçuzeiro (Theobroma grandiflorum) cultivado em solução nutritiva. Sci. Agric., 5:407-414, 1994.

SILVA JUNIOR, J.P. \& CARDOSO, E.J.B.N. Micorriza arbuscular em cupuaçu e pupunha cultivados em sistema agroflorestal e em monocultivo na Amazônia central. Pesq. Agropec. Bras.,.41:819-825, 2006.

URANO, E.O.M.; KURIHARA, C.H.; MAEDA, S.; VITORINO, A.C.T.; GONÇALVES, M.C. \& MARCHETTI, M.E. Determinação de teores ótimos de nutrientes em soja pelos métodos Chance Matemática, sistema integrado de diagnose e recomendação e diagnose da composição nutricional. $R$. Bras. Ci. Solo, 31:63-72, 2007. 
WADT, P.G.S.; NOVAIS, R.F.; ALVAREZ V, V.H.; FONSECA, S. \& BARROS, N.F. Valores de referência para macronutrientes em eucalipto obtidos pelos métodos Dris e chance matemática. R. Bras. Ci. Solo, 22:685-692, 1998a.

WADT, P.G.S. Relationships between soil class and nutritional status of coffee crops. R. Bras. Ci. Solo, 29:227-234, 2005.

WADT, P.G.S.; ALVAREZ V, V.H.; NOVAIS, R.F.; FONSECA, S.; BARROS, N.F. O método da Chance Matemática na interpretação de dados de levantamento nutricional de eucalipto. R. Bras. Ci. Solo, 22:773-778, 1998b.
WADT, P.G.S.; SILVA, D.J.; MAIA, C.E.; TOMÉ JUNIOR, J.B.; PINTO, P.A.C. \& MACHADO, P.L.O.A. Modelagem de funções no cáculo dos índices DRIS. Pesq. Agropec. Bras., 42:57-64, 2007.

YUYAMA, K.; PEREIRA, B.G.; LIMA, F.S.F. \& ANJOS, J.S.S. Efeito da correção e adubação do solo no desenvolvimento da planta de cupuaçu, cultivada a pleno sol, na Amazônia central. In: CONGRESSO BRASILEIRO DE FRUTICULTURA, 20., Vitória, 2008. Resumos... Vitória, 2008. 6p. 
